\title{
Block Method for the Solution of First Order Nonlinear ODEs and Its Application to HIV Infection of $\mathrm{CD4}^{+}$T Cells Model
}

\author{
Adeyeye Oluwaseun*, Omar Zurni \\ Department of Mathematics \& Statistics, School of Quantitative Sciences, Malaysia
}

Received November 18, 2020; Revised March 7, 2021; Accepted March 19, 2021

\section{Cite This Paper in the following Citation Styles}

(a): [1] Adeyeye Oluwaseun, Omar Zurni, "Block Method for the Solution of First Order Nonlinear ODEs and Its Application to HIV Infection of $\mathrm{CD}^{+}$T Cells Model," Mathematics and Statistics, Vol. 9, No. 3, pp. 371 - 378, 2021. DOI: 10.13189/ms.2021.090319.

(b): Adeyeye Oluwaseun, Omar Zurni (2021). Block Method for the Solution of First Order Nonlinear ODEs and Its Application to HIV Infection of $\mathrm{CD}^{+} \mathrm{T}$ Cells Model. Mathematics and Statistics, 9(3), 371 - 378. DOI: 10.13189/ms.2021.090319.

Copyright $\subseteq 2021$ by authors, all rights reserved. Authors agree that this article remains permanently open access under the terms of the Creative Commons Attribution License 4.0 International License

\begin{abstract}
Some of the issues relating to the human immunodeficiency virus (HIV) epidemic can be expressed as a system of nonlinear first order ordinary differential equations. This includes modelling the spread of the HIV virus in infecting $\mathrm{CD} 4+\mathrm{T}$ cells that help the human immune system to fight diseases. However, real life differential equation models usually fail to have an exact solution, which is also the case with the nonlinear model considered in this article. Thus, an approximate method, known as the block method, is developed to solve the system of first order nonlinear differential equation. To develop the block method, a linear block approach was adopted, and the basic properties required to classify the method as convergent were investigated. The block method was found to be convergent, which ascertained its usability for the solution of the model. The solution obtained from the newly developed method in this article was compared to previous methods that have been adopted to solve same model. In order to have a justifiable basis of comparison, two-step length values were substituted to obtain a one-step and two-step block method. The results show the newly developed block method obtaining accurate results in comparison to previous studies. Hence, this article has introduced a new method suitable for the direct solution of first order differential equation models without the need to simplify to a system of linear algebraic equations. Likewise, its convergent properties and accuracy also give the block method an edge over existing methods.
\end{abstract}

Keywords HIV Infection, CD4 $4^{+} \mathrm{T}$ Cells, Numerical, Block Method, First Order

\section{Introduction}

The recent trend in research has delved into mathematical modelling of real-life cases, and problems existing in the field of biological sciences have not been left out. One of the major issues which are still being investigated intensely is the human immunodeficiency virus (HIV). In the investigation by [1], it was stated that an estimated number of 35 million adults worldwide are HIV positive. The $\mathrm{CD}^{+} \mathrm{T}$ cells in human body, also known as leukocytes or $\mathrm{T}$ helper cells, are quite vital to the immune system, as it joins other cells in the human immune system to fight diseases. The effect of the HIV on $\mathrm{CD} 4^{+} \mathrm{T}$ cells is that it depletes and infects these cells, thus most important to the production of acquired immunodeficiency syndrome (AIDS). This development from HIV to AIDS happens due to the inability of human body to defend itself against other infections as the $\mathrm{CD}^{+}{ }^{+} \mathrm{T}$ cells are destroyed in the blood. However, if the virus is detected on time and treated, the human immune system can still be protected and hence controlling the spread or growth of the HIV by determining which $\mathrm{CD} 4^{+} \mathrm{T}$ cells are infected, and which are not [2]. 
[3] developed an HIV infection of the human immune system model of three variables that include the population sizes of the infected cells, the uninfected cells and the free particles of the virus. Further study [4] expanded the paradigm in a manner that replicates various of the scientifically acquired signs of AIDS. Four factors consisting of latently infected cells, uninfected cells, free particles of viruses and actively infected cells were considered in this expansion. However, it was considered by [5] that the assumption should follow that all the infected cells are able to produce the virus, thus defining the dynamic model required to observe the $\mathrm{CD} 4^{+} \mathrm{T}$ cells, as a system of three nonlinear ordinary differential equations defined as:

$$
\left\{\begin{array}{c}
\frac{d T(t)}{d t}=\gamma_{1}-\gamma_{2} T(t)+\gamma_{3} T(t)\left(1-T_{\max }{ }^{-1}(T(t)+I(t))\right)-\gamma_{4} V(t) T(t), \\
\frac{d I(t)}{d t}=\gamma_{4} V(t) T(t)-\gamma_{5} I(t), \\
\frac{d V(t)}{d t}=N \gamma_{5} I(t)-\gamma_{6} V(t) .
\end{array}\right.
$$

In Equation (1), the concentration of susceptible CD4+T cells, CD4+T cells infected with HIV viruses and free HIV virus particles in the blood at time t, denoted as, and respectively, are the three basic component models. The definition of each parameter in Equation (1) is shown

\begin{tabular}{|c|c|}
\hline Parameter & Description \\
\hline$\gamma_{1}$ & Level of body-produced $\mathrm{CD}^{+} \mathrm{T}$ cells \\
\hline$\gamma_{2}$ & Normal rates of uninfected T cells' turnover \\
\hline$\gamma_{3}$ & $\mathrm{CD}^{+} \mathrm{T}$ cell growth rate \\
\hline$\gamma_{4}$ & Level of infection \\
\hline$\gamma_{5}$ & Infected T cells' normal turnover rate \\
\hline$\gamma_{6}$ & Normal turnover rate of particles from viruses \\
\hline$T_{\max }$ & $\begin{array}{l}\text { In the body, the maximum } \mathrm{CD}^{+} \mathrm{T} \text { cell } \\
\text { concentration }\end{array}$ \\
\hline$N$ & $\begin{array}{l}\text { Particles of viruses manufactured by } \\
\text { contaminated } \mathrm{CD}^{+} \mathrm{T} \text { cells }\end{array}$ \\
\hline
\end{tabular}
in Table 1, as described by [5].

Table 1. Parameters of the HIV $\mathrm{CD}^{+}{ }^{+} \mathrm{T}$ cells model [2]

Over the years, many studies have considered solutions to the model in Equation (1). These include [6] the suggestion of an updated variational iteration method, [7] the implementation of the Bessel collocation method, [8] the adoption of the Laplace Adomian decomposition method, [9] the use of the differential transformation method, [10] the introduction of the multi-stage Laplace Adomian decomposition method, and [11] the suggestion of the perturbation iteration algorithm. More recent investigations to obtain a numerical solution to the system of nonlinear ordinary differential equations have been explored by [2] with the use of the Shifted-Lagrangian
Jacobi polynomials, [12] adopting a collocation method based on Bernoulli polynomials, [13] providing the model's approximate solution using orthonormal Bernstein polynomials, [14] developing a novel biologically-inspired computing framework, [15] combining the traditional homotopy analysis method with the Laplace transformation approach, among others. In general, the definition adopted by these authors was a reduction to the corresponding system of nonlinear algebraic equations of the nonlinear system of ordinary differential equations, which is then rectified by following some reasonable computational solution, such as the well-known method of Newton. This approach of the reduction which involves more computations can be bypassed with the application of direct methods for solving ordinary differential equations, such as the block method.

The vast applicability of the block method alongside its impressive accuracy in terms of evaluation to the accurate solutions of first order ODE models, has been documented in various studies [16-23]. However, the application of the block method to solve dynamic model required to examine the $\mathrm{CD}^{+}{ }^{+} \mathrm{T}$ cells in this article is quite uncommon, asides the recent study by [24] who proposed a separately diagonally implicit block backward differentiation formula designed for order two to approximate the solution to the model in Equation (1). Whereas, the solution of the model in this article will consider the use of two block methods for first order differential equations with impressive accuracy, despite their order, in comparison to previous solutions in literature.

\section{Methodology}

This section details the derivation of the block methods in addition to discussing their individual convergence properties. Deriving the block methods to be utilised as a new approach to numerically solve HIV infection model of $\mathrm{CD}^{+}{ }^{+} \mathrm{T}$ cells involves considering the block method scheme of the form

$$
\begin{aligned}
& T_{n+\xi}=\sum_{i=0}^{m-1} \frac{(\xi h)^{i}}{i !} T_{n}^{(i)}+\sum_{i=0}^{k} \varphi_{T i \xi}\left(\gamma_{1}-\gamma_{2} T_{n+i}+\gamma_{3} T_{n+i}\left(1-T_{\max }{ }^{-1}\left(T_{n+i}+I_{n+i}\right)\right)-\gamma_{4} V_{n+i+1} T_{n+i}\right) \\
& I_{n+\xi}=\sum_{i=0}^{m-1} \frac{(\xi h)^{i}}{i !} I_{n}^{(i)}+\sum_{i=0}^{k} \varphi_{n i \xi}\left(\gamma_{4} V_{n+i} T_{n+i}-\gamma_{5} I_{n+i}\right) \\
& V_{n+\xi}=\sum_{i=0}^{m-1} \frac{(\xi h)^{i}}{i !} V_{n}^{(i)}+\sum_{i=0}^{k} \varphi_{n i \xi}\left(N \gamma_{5} I_{n+i}-\gamma_{6} V_{n+i}\right)
\end{aligned}
$$

and also

$$
\begin{aligned}
& T_{n+\xi}=\sum_{i=0}^{m-1} \frac{(\xi h)^{i}}{i !} T_{n}^{(i)}+\sum_{i=0}^{k} \phi_{T i \xi}\left(\gamma_{1}-\gamma_{2} T_{n+i}+\gamma_{3} T_{n+i}\left(1-T_{\max }{ }^{-1}\left(T_{n+i}+I_{n+i}\right)\right)-\gamma_{4} V_{n+i} T_{n+i}\right) \\
& I_{n+\xi}=\sum_{i=0}^{m-1} \frac{(\xi h)^{i}}{i !} I_{n}^{(i)}+\sum_{i=0}^{k} \phi_{n i \xi}\left(\gamma_{4} V_{n+i} T_{n+i}-\gamma_{5} I_{n+i}\right) \\
& V_{n+\xi}=\sum_{i=0}^{m-1} \frac{(\xi h)^{i}}{i !} V_{n}^{(i)}+\sum_{i=0}^{k} \phi_{n i \xi}\left(N \gamma_{5} I_{n+i}-\gamma_{6} V_{n+i}\right)
\end{aligned}
$$


The block scheme is presented as a system of equations to conform with the model as defined in equation (1). In Equations (2) and (3), $m$ is the order of the differential equation model, $k$ denotes the step number chosen as 1 in Equation (2) and 2 in Equation (3), with $\xi=1,2, \ldots, k$.

These $k$ values were selected in order to obtain suitable block methods of equal or lower order to the other previously developed numerical methods for comparison. Following the linear block approach by [25], the unknown coefficients for the $k=1$ block method are derived from the expression

$$
\begin{aligned}
& \varphi_{j i \xi}=A_{j}^{-1} B_{j}, j=T, I, V \quad \text { with } A_{j}=\left(\begin{array}{ll}
1 & 1 \\
0 & h
\end{array}\right) \text { and } \\
& B_{j}=\left(\begin{array}{c}
(\xi h) \\
\frac{(\xi h)^{2}}{2 !}
\end{array}\right)
\end{aligned}
$$

while the coefficients for the $k=2$ block method are derived from the expression

$$
\begin{aligned}
\phi_{j i \xi} & =A_{j}^{-1} B_{j}, j=T, I, V \text { with } A_{j}=\left(\begin{array}{ccc}
1 & 1 & 1 \\
0 & h & 2 h \\
0 & \frac{(h)^{2}}{2 !} & \frac{(2 h)^{2}}{2 !}
\end{array}\right) \text { and } \\
B_{j} & =\left(\begin{array}{c}
(\xi h) \\
\frac{(\xi h)^{2}}{2 !} \\
\frac{(\xi h)^{3}}{3 !}
\end{array}\right) \text {. }
\end{aligned}
$$

This implies that, for the one-step block method, Equation (2) takes the form

$$
\begin{aligned}
& T_{n+1}=T_{n}+\varphi_{T 01} \chi_{T 0}+\varphi_{T 11} \chi_{T 1} \\
& I_{n+1}=I_{n}+\varphi_{I 01} \chi_{I 0}+\varphi_{I 11} \chi_{I 1} \\
& V_{n+1}=V_{n}+\varphi_{V 01} \chi_{V 0}+\varphi_{V 11} \chi_{V 1}
\end{aligned}
$$

while Equation (3) takes the form

$$
\begin{aligned}
& T_{n+1}=T_{n}+\phi_{T 01} \chi_{T 0}+\phi_{T 11} \chi_{T 1}+\phi_{T 21} \chi_{T 2} \\
& T_{n+2}=T_{n}+\phi_{T 02} \chi_{T 0}+\phi_{T 12} \chi_{T 1}+\phi_{T 22} \chi_{T 2} \\
& I_{n+1}=I_{n}+\phi_{I 01} \chi_{I 0}+\phi_{I 11} \chi_{I 1}+\phi_{I 21} \chi_{I 2} \\
& I_{n+2}=I_{n}+\phi_{I 02} \chi_{T 0}+\phi_{I 12} \chi_{T 1}+\phi_{I 22} \chi_{I 2} \\
& V_{n+1}=V_{n}+\phi_{V 01} \chi_{V 0}+\phi_{V 11} \chi_{V 1}+\phi_{V 21} \chi_{V 2} \\
& V_{n+2}=V_{n}+\phi_{V 02} \chi_{V 0}+\phi_{V 12} \chi_{V 1}+\phi_{V 22} \chi_{V 2}
\end{aligned}
$$

where

$$
\begin{aligned}
& \chi_{T 0}=\gamma_{1}-\gamma_{2} T_{n}+\gamma_{3} T_{n}\left(1-T_{\max }{ }^{-1}\left(T_{n}+I_{n}\right)\right)-\gamma_{4} V_{n} T_{n} \\
& \chi_{T 1}=\gamma_{1}-\gamma_{2} T_{n+1}+\gamma_{3} T_{n+1}\left(1-T_{\max }{ }^{-1}\left(T_{n+1}+I_{n+1}\right)\right)-\gamma_{4} V_{n+1} T_{n+1} \\
& \chi_{T 2}=\gamma_{1}-\gamma_{2} T_{n+2}+\gamma_{3} T_{n+2}\left(1-T_{\max }{ }^{-1}\left(T_{n+2}+I_{n+2}\right)\right)-\gamma_{4} V_{n+2} T_{n+2} \\
& \chi_{I 0}=\gamma_{4} V_{n} T_{n}-\gamma_{5} I_{n} \\
& \chi_{I 1}=\gamma_{4} V_{n+1} T_{n+1}-\gamma_{5} I_{n+1} \\
& \chi_{I 2}=\gamma_{4} V_{n+2} T_{n+2}-\gamma_{5} I_{n+2} \\
& \chi_{V 0}=N \gamma_{5} I_{n}-\gamma_{6} V_{n} \\
& \chi_{V 1}=N \gamma_{5} I_{n+1}-\gamma_{6} V_{n+1} \\
& \chi_{V 2}=N \gamma_{5} I_{n+2}-\gamma_{6} V_{n+2}
\end{aligned}
$$

Hence,

$$
\left(\begin{array}{l}
\varphi_{T 01} \\
\varphi_{T 11}
\end{array}\right)=\left(\begin{array}{ll}
1 & 1 \\
0 & h
\end{array}\right)^{-1}\left(\begin{array}{c}
h \\
\frac{(h)^{2}}{2 !}
\end{array}\right)=\left(\begin{array}{c}
\frac{h}{2} \\
\frac{h}{2}
\end{array}\right),
$$

$$
\begin{gathered}
\left(\begin{array}{l}
\phi_{T 01} \\
\phi_{T 11} \\
\phi_{T 21}
\end{array}\right)=\left(\begin{array}{ccc}
1 & 1 & 1 \\
0 & h & 2 h \\
0 & \frac{(h)^{2}}{2 !} & \frac{(2 h)^{2}}{2 !}
\end{array}\right)^{-1}\left(\begin{array}{c}
h \\
\frac{(h)^{2}}{2 !} \\
\frac{(h)^{3}}{3 !}
\end{array}\right)=\left(\begin{array}{c}
\frac{5 h}{12} \\
\frac{2 h}{3} \\
-\frac{h}{12}
\end{array}\right) \text { and } \\
\left(\begin{array}{l}
\phi_{T 02} \\
\phi_{T 12} \\
\phi_{T 22}
\end{array}\right)=\left(\begin{array}{ccc}
1 & 1 & 1 \\
0 & h & 2 h \\
0 & \frac{(h)^{2}}{2 !} & \frac{(2 h)^{2}}{2 !}
\end{array}\right)^{-1}\left(\begin{array}{c}
2 h \\
\frac{(2 h)^{2}}{2 !} \\
\frac{(2 h)^{3}}{3 !}
\end{array}\right)=\left(\begin{array}{c}
\frac{h}{3} \\
\frac{4 h}{3} \\
\frac{h}{3}
\end{array}\right) .
\end{gathered}
$$

Similarly, $\left(\varphi_{I 01}, \varphi_{I 11}\right)^{T}=\left(\varphi_{V 01}, \varphi_{V 11}\right)^{T}=\left(\frac{h}{2}, \frac{h}{2}\right)^{T}$,

$$
\begin{gathered}
\left(\phi_{I 01}, \phi_{I 11}, \phi_{I 21}\right)^{T}=\left(\phi_{V 01}, \phi_{V 11}, \phi_{V 21}\right)^{T}=\left(\frac{5 h}{12}, \frac{2 h}{3},-\frac{h}{12}\right)^{T} \\
\text { and }\left(\phi_{I 02}, \phi_{I 12}, \phi_{I 22}\right)^{T}=\left(\phi_{V 02}, \phi_{V 12}, \phi_{V 22}\right)^{T}=\left(\frac{h}{3}, \frac{4 h}{3}, \frac{h}{3}\right)^{T} \text {. }
\end{gathered}
$$

Substituting the obtained $\varphi_{j i \xi}, \phi_{j i \xi}$-values back in Equations (4) and (5) gives the required block methods to solve the model as defined in equation (1).

Now, considering the convergence properties of the block methods in Equations (2) and (3). For these block methods to be convergent, they must satisfy the property of linear multistep system $\mathrm{s}$ which is consistent and zero-stable [26].

Firstly, the consistency of the methods is checked, where a linear multistep system is constant if it has an order of $p \geq 1$. Collectively merging all the variables in the model rewrites the block expressions as: 


$$
\begin{gathered}
j_{n+1}=j_{n}+\varphi_{j 01} \chi_{j 0}+\varphi_{j 11} \chi_{j 1} \\
j_{n+1}=j_{n}+\phi_{j 01} \chi_{j 0}+\phi_{j 11} \chi_{j 1}+\phi_{j 21} \chi_{j 2} \\
j_{n+2}=j_{n}+\phi_{j 02} \chi_{j 0}+\phi_{j 12} \chi_{j 1}+\phi_{j 22} \chi_{j 2}
\end{gathered}
$$

with $j=T, I, V$. The individual terms $j_{n}, j_{n+1}$, and $j_{n+2}$ in Equations (7)- (9), including terms within the previously defined $\chi_{j 0}, \chi_{j 1}$, and $\chi_{j 2}$, are expanded using Taylor series expansion about $t=t_{n}$. Let $C_{m}$ be the value obtained on equating coefficients of $h^{m} j^{(m)}\left(t_{n}\right)$ in Equations (7)-(9). Equation (7) has $C_{0}=C_{1}=C_{2}=0, C_{3}=-\frac{h^{3}}{12}$, while in Equation (8), $C_{0}=C_{1}=C_{2}=C_{3}=0, C_{4}=\frac{h^{4}}{24}$, and for Equation (9), $C_{0}=C_{1}=C_{2}=C_{3}=0, C_{4}=0, C_{5}=-\frac{h^{5}}{90}$. Following the definition by [27], a linear multistep system for solving first order differential equations is of order $p$ if $C_{0}=C_{1}=C_{2}=\ldots=C_{p}$ and $C_{p+1} \neq 0$. This implies that the block methods are consistent as the order $p=\{2,3,4\}$ satisfies $p \geq 1$.

Considering the second condition for convergence, this is the zero-stability criterion. The block method in the subsequent matrix difference equation form

$$
A^{0} j_{n+k}=A^{1} j_{n-k}+h\left[B^{0} j_{n+k}^{(1)}+B^{1} j_{n-k}^{(1)}\right]
$$

is zero-stable if the characteristic polynomial takes the form

$$
\rho(R)=\operatorname{det}\left(R_{i j} A^{0}-A^{1}\right) ; \quad R_{i j}=R^{i} \delta_{i j} \quad(i=1,2, \ldots, k)
$$

and the roots of $\rho(R)=0$ satisfies the root condition that every its roots lie in or on the unit circle, with those on the boundary being simple, that is, all roots satisfy $|R| \leq 1, j=1, \ldots, k \quad, \quad$ and a few that satisfy $|R|=1, j=1, \ldots, k$, are common, where $v$ is said to be a simple root of $\rho(R)$ if $(v-R)$ is a factor, but $(v-R)^{2}$ is not. The coefficients $A^{0}$ and $A^{1}$ of the matrix difference equation form for the block methods are substituted in Equation (11) to obtain the characteristic polynomial $\rho(R)$ as $R-1$ and $R^{3}-R$, for the one-step and two-step block methods respectively. The absolute value of the roots of $\rho(R)$ gives values of 0 and 1 . Therefore, all the roots satisfy $|R| \leq 1$ and the block methods are zero-stable. Hence, since the block methods have satisfied both conditions of consistency and zero-stability, this implies that both block methods are convergent.

\section{Results}

In this section, the block methods will be adopted to solve the dynamic model that deals with the HIV infection of $\mathrm{CD} 4^{+} \mathrm{T}$ cells as defined in Equation (1). In the absence of the exact solution, comparison will be made to the converged solution obtained by [2] and [12]. The conditions at the initial point for the model in Equation (1) are $T(0)=0.1, I(0)=0$ and $V(0)=0.1$ with the unknown parameters set as $\gamma_{1}=0.1, \gamma_{2}=0.02, \gamma_{3}=3$, $\gamma_{4}=0.0027, \quad \gamma_{5}=0.3, \quad \gamma_{6}=2.4, T_{\max }=1500, \quad N=10$ The tables detailing the results obtained demonstrate the numerical value of $T(t), I(t)$ and $V(t)$ within the interval $[0,1]$ in comparison to recent studies exploring an estimated solution to the model.

Table 2. Numerical results for $T(t)$

\begin{tabular}{ccccccc}
\hline$t$ & $\begin{array}{c}\text { Converged } \\
\text { Solution }\end{array}$ & $\begin{array}{c}\text { Computed Solution } \\
{[\mathbf{2 8}]}\end{array}$ & $\begin{array}{c}\text { Computed Solution } \\
{[\mathbf{2 4}]}\end{array}$ & $\begin{array}{c}\text { Computed Solution } \\
{[\mathbf{1 3}]}\end{array}$ & $\begin{array}{c}\text { One-Step Block } \\
\text { Method }\end{array}$ & $\begin{array}{c}\text { Two-Step Block } \\
\text { Method }\end{array}$ \\
\hline 0.1 & - & - & - & - & 0.1463630478 & 0.1463590814 \\
0.2 & 0.2088080843 & 0.2073594783 & 0.2090205532 & 0.2129281262 & 0.2088187668 & 0.2088080818 \\
0.3 & - & - & - & - & 0.2929509957 & 0.2929294089 \\
0.4 & 0.4062405428 & 0.4034685968 & 0.4065545236 & 0.4110116992 & 0.4062792966 & 0.4062405336 \\
0.5 & - & - & - & - & 0.5589285924 & 0.5588633428 \\
0.6 & 0.7644238985 & 0.7595171444 & 0.7648420298 & 0.7757846339 & 0.7645293020 & 0.7644238736 \\
0.7 & - & - & - & - & 1.0414263729 & 1.0412607830 \\
0.8 & 1.4140468519 & 1.4050224494 & 1.4145067965 & 1.4347539521 & 1.4143015118 & 1.4140467917 \\
0.9 & - & - & - & - & 1.9163467361 & 1.9159611404 \\
1.0 & 2.5915948517 & 2.5753421941 & 2.5840259154 & 2.7432245704 & 2.5921710130 & 2.5915947158 \\
\hline
\end{tabular}


Table 3. Absolute error (AE) results of computed $T(t)$ solutions with converged solution

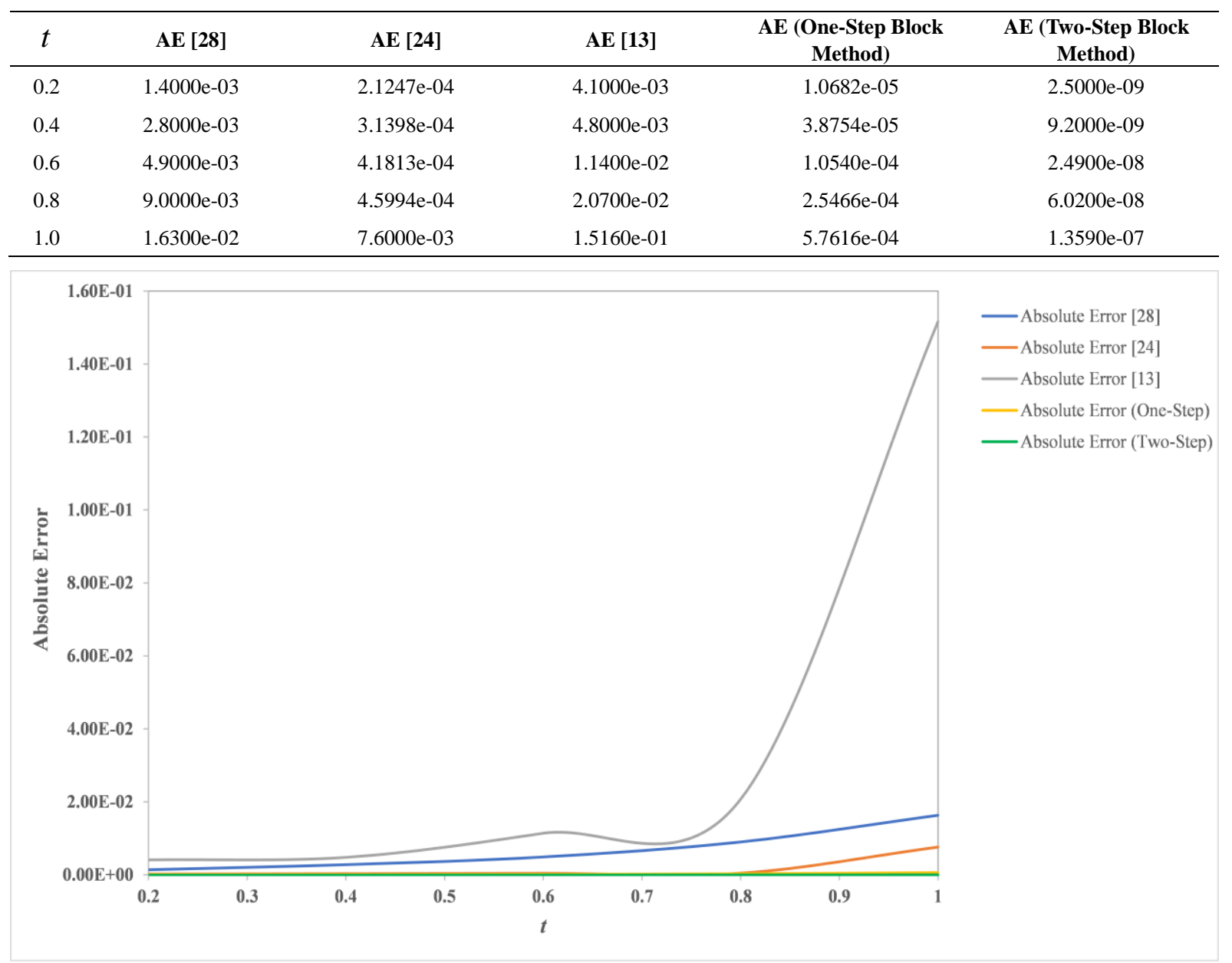

Figure 1. Absolute error (AE) results of computed $T(t)$

Table 4. Numerical results for $I(t)$

\begin{tabular}{ccccccc}
\hline$t$ & $\begin{array}{c}\text { Converged } \\
\text { Solution }\end{array}$ & $\begin{array}{c}\text { Computed } \\
\text { Solution [28] }\end{array}$ & $\begin{array}{c}\text { Computed } \\
\text { Solution [24] }\end{array}$ & $\begin{array}{c}\text { Computed } \\
\text { Solution [13] }\end{array}$ & $\begin{array}{c}\text { One-Step Block } \\
\text { Method }\end{array}$ & $\begin{array}{c}\text { Two-Step Block } \\
\text { Method }\end{array}$ \\
\hline 0.1 & - & - & - & - & 0.0000028649 & 0.0000028649 \\
0.2 & 0.0000060327 & 0.0000093532 & 0.0000060459 & 0.0000059037 & 0.0000060327 & 0.0000060327 \\
0.3 & - & - & - & - & 0.0000094715 & 0.0000094714 \\
0.4 & 0.0000131583 & 0.0000108470 & 0.0000131824 & 0.0000129974 & 0.0000131586 & 0.0000131583 \\
0.5 & - & - & - & - & 0.0000170793 & 0.0000170787 \\
0.6 & 0.0000212238 & 0.0000207830 & 0.0000212573 & 0.0000212334 & 0.0000212246 & 0.0000212238 \\
0.7 & - & - & - & - & 0.0000255910 & 0.0000255898 \\
0.8 & 0.0000301774 & 0.0000266389 & 0.0000302185 & 0.0000302700 & 0.0000301790 & 0.0000301774 \\
0.9 & - & - & - & - & 0.0000349929 & 0.0000349909 \\
1.0 & 0.0000400378 & 0.0000388399 & 0.0000399547 & 0.0000394304 & 0.0000400404 & 0.0000400378 \\
\hline
\end{tabular}

Table 5. Absolute error (AE) results of computed $I(t)$ solutions with converged solution

\begin{tabular}{cccccc}
\hline$t$ & AE [28] & AE [24] & AE [13] & $\begin{array}{c}\text { AE (One-Step Block } \\
\text { Method) }\end{array}$ & $\begin{array}{c}\text { AE (Two-Step Block } \\
\text { Method) }\end{array}$ \\
\hline 0.2 & $3.3205 \mathrm{e}-06$ & $1.3200 \mathrm{e}-08$ & $1.2900 \mathrm{e}-07$ & $0.0000 \mathrm{e}+00$ & $0.0000 \mathrm{e}+00$ \\
0.4 & $2.3113 \mathrm{e}-06$ & $2.4100 \mathrm{e}-08$ & $1.6090 \mathrm{e}-07$ & $3.0000 \mathrm{e}-10$ & $0.0000 \mathrm{e}+00$ \\
0.6 & $4.4080 \mathrm{e}-07$ & $3.3500 \mathrm{e}-08$ & $9.6000 \mathrm{e}-09$ & $8.0000 \mathrm{e}-10$ & $0.0000 \mathrm{e}+00$ \\
0.8 & $3.5385 \mathrm{e}-06$ & $4.1100 \mathrm{e}-08$ & $9.2600 \mathrm{e}-08$ & $1.6000 \mathrm{e}-09$ & $0.0000 \mathrm{e}+00$ \\
1.0 & $1.1979 \mathrm{e}-06$ & $8.3100 \mathrm{e}-08$ & $6.0740 \mathrm{e}-07$ & $2.6000 \mathrm{e}-09$ & $0.0000 \mathrm{e}+00$ \\
\hline
\end{tabular}


Its Application to HIV Infection of $\mathrm{CD}^{+}{ }^{+} \mathrm{T}$ Cells Model

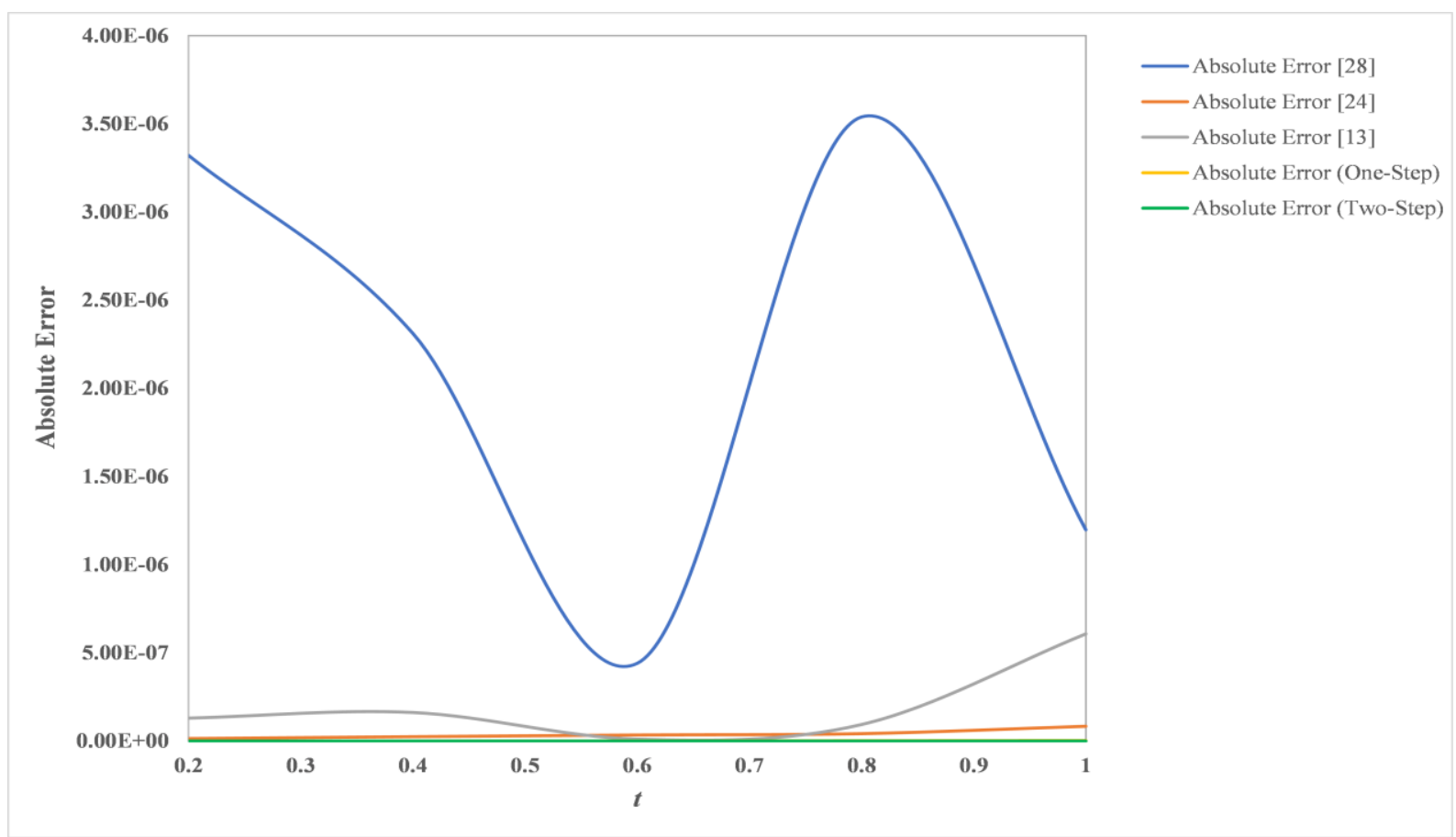

Figure 2. Absolute error (AE) results of computed $I(t)$

Table 6. Numerical results for $V(t)$

\begin{tabular}{ccccccc}
\hline$t$ & $\begin{array}{c}\text { Converged } \\
\text { Solution }\end{array}$ & $\begin{array}{c}\text { Computed } \\
\text { Solution [28] }\end{array}$ & $\begin{array}{c}\text { Computed } \\
\text { Solution [24] }\end{array}$ & $\begin{array}{c}\text { Computed } \\
\text { Solution [13] }\end{array}$ & $\begin{array}{c}\text { One-Step Block } \\
\text { Method }\end{array}$ & $\begin{array}{c}\text { Two-Step Block } \\
\text { Method }\end{array}$ \\
\hline 0.1 & - & - & - & - & 0.0786622699 & 0.0786631764 \\
0.2 & 0.0618798432 & 0.0618799249 & 0.0619225726 & 0.0616038027 & 0.0618784173 & 0.0618798434 \\
0.3 & - & - & - & - & 0.0486768065 & 0.0486784892 \\
0.4 & 0.0382948878 & 0.0382947904 & 0.0383177394 & 0.0381135846 & 0.0382931232 & 0.0382948880 \\
0.5 & - & - & - & - & 0.0301261389 & 0.0301278741 \\
0.6 & 0.0237045500 & 0.0237043306 & 0.0237164782 & 0.0236278850 & 0.0237029125 & 0.0237045503 \\
0.7 & - & - & - & - & 0.0186514182 & 0.0186529210 \\
0.8 & 0.0146803637 & 0.0146803895 & 0.0146863853 & 0.0146194704 & 0.0146790131 & 0.0146803639 \\
0.9 & - & - & - & - & 0.0115554996 & 0.0115566945 \\
1.0 & 0.0091008440 & 0.0091008815 & 0.0091490338 & 0.0081082206 & 0.0090998013 & 0.0091008452 \\
\hline
\end{tabular}

Table 7. Absolute error (AE) results of computed $V(t)$ solutions with converged solution

\begin{tabular}{cccccc}
\hline$t$ & AE [28] & AE [24] & AE [13] & $\begin{array}{c}\text { AE (One-Step Block } \\
\text { Method) }\end{array}$ & $\begin{array}{c}\text { AE (Two-Step Block } \\
\text { Method) }\end{array}$ \\
\hline 0.2 & $8.1700 \mathrm{e}-08$ & $4.2729 \mathrm{e}-05$ & $2.7604 \mathrm{e}-04$ & $1.4259 \mathrm{e}-06$ & $2.0000 \mathrm{e}-10$ \\
0.4 & $9.7400 \mathrm{e}-08$ & $2.2852 \mathrm{e}-05$ & $1.8130 \mathrm{e}-04$ & $1.7646 \mathrm{e}-06$ & $2.0000 \mathrm{e}-10$ \\
0.6 & $2.1940 \mathrm{e}-07$ & $1.1928 \mathrm{e}-05$ & $7.6665 \mathrm{e}-05$ & $1.6375 \mathrm{e}-06$ & $3.0000 \mathrm{e}-10$ \\
0.8 & $2.5800 \mathrm{e}-08$ & $6.0216 \mathrm{e}-06$ & $6.0893 \mathrm{e}-05$ & $1.3506 \mathrm{e}-06$ & $2.0000 \mathrm{e}-10$ \\
1.0 & $3.7500 \mathrm{e}-08$ & $4.8190 \mathrm{e}-05$ & $9.9262 \mathrm{e}-04$ & $1.0427 \mathrm{e}-06$ & $1.2000 \mathrm{e}-09$ \\
\hline
\end{tabular}




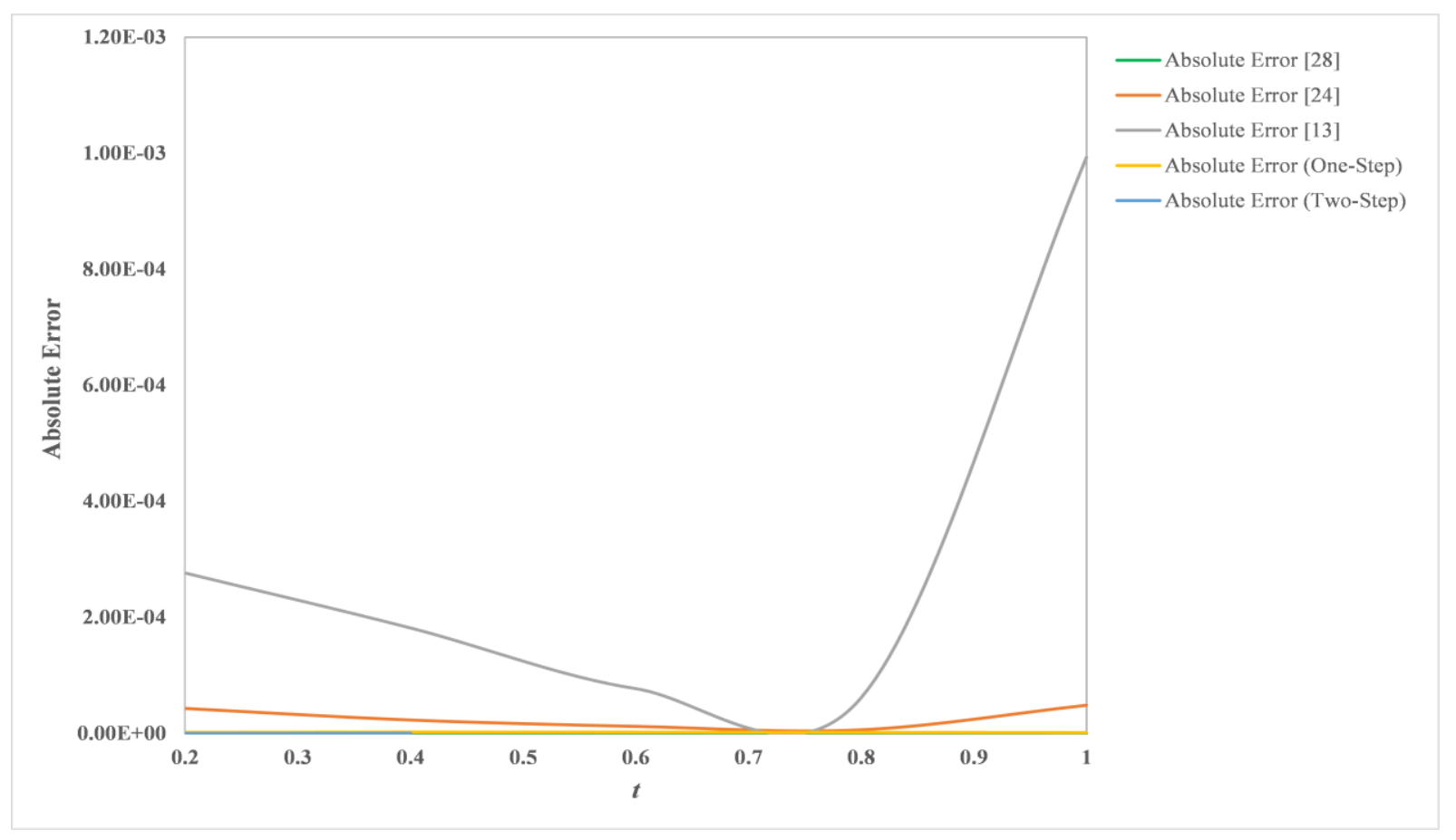

Figure 3. Absolute error (AE) results of computed $V(t)$

\section{Conclusions}

This article has developed one and two-step block methods for the solution of a system of first order nonlinear ordinary differential equations. The properties of the methods as shown in the methodology section have defined the methods to be of low orders, hence the expectation of low accuracy. Rather, the block methods have displayed impressive performance in comparison to results from previous studies. The methods that were compared to the one and two-step block methods, as shown in Tables 2-7 include the optimization method by [28] which is based on generalized polynomials of degrees 5 and 7 , the order 2 singly diagonally implicit backward block method by [24], and [13] collocation method implemented with degree 8 orthonormal Bernstein polynomials. The lower and equal order of the block methods in this article gives a good basis of comparison to these selected studies. As seen in Table 2, Table 4, and Table 6 , the previous studies presented their results for $t=[0: 0.2: 1]$, while the solutions from the block methods were shown for $t=[0: 0.1: 1]$, which gives room for better comparison for future studies. When considering the absolute error values as shown in Table 3, Table 5, and Table 7, the one-step method shows improved accuracy than the method having same order 2 as itself in Table 3 and Table 5. Likewise, Figure 1, Figure 2, and Figure 3 clearly show the block method having absolute error tending to zero. In general, the two block methods performed than the other approaches for the solutions of $T(t)$ and $I(t)$, particularly the two-step block method which obtained the same values as the converged solution of $I(t)$ despite having low order. For the solution for $V(t)$, the absolute error values in Table 7 shows the one-step method is still performing better than the order 2 method of [24], with the method by [28] showing good accuracy but the two-step block method gave the most accurate results in terms of absolute error comparison. This has shown that the new block methods, despite being methods of low order perform favourably when adopted for the solution of a system of nonlinear first order ordinary differential equations, which can be sued in modelling various real-life scenarios, including the model for HIV Infection of $\mathrm{CD}^{+} \mathrm{T}$ considered in this article.

\section{REFERENCES}

[1] Monaco, C. L., Gootenberg, D. B., Zhao, G., Handley, S. A., Ghebremichael, M. S., Lim, E. S., Lankowski, A., Baldridge, M. T., Wilen, C. B., Flagg, M., Norman, J. M., Keller B. C., Luévano, J. M., Wang, D., Boum, Y., Martin, J. N., Hunt, P. W., Bangsberg, D. R., Siedner, M. J., Kwon, D. S., and Virgin, H. W. "Altered virome and bacterial microbiome in human immunodeficiency virus-associated acquired immunodeficiency syndrome." Cell Host and Microbe 19, no. 3 (2016): 311-322.

[2] Parand, K., Latifi, S., and Moayeri, M. M. "Shifted Lagrangian Jacobi collocation scheme for numerical solution of a model of HIV infection."SeMA Journal 75, no. 3(2018): 379-398.

[3] Perelson, A. S. (1989). Modeling the interaction of the immune system with HIV. In Mathematical and statistical approaches to AIDS epidemiology (pp. 350-370). Springer, 
Berlin, Heidelberg.

[4] Perelson, A. S., Kirschner, D. E., and De Boer, R. "Dynamics of HIV infection of CD4 ${ }^{+} \mathrm{T}$ cells." Mathematical Biosciences 114, no. 1(1993): 81-125.

[5] Culshaw, R. V., and Ruan, S. "A delay-differential equation model of HIV infection of $\mathrm{CD}^{+} \mathrm{T}$-cells." Mathematical Biosciences 165, no. 1(2000): 27-39.

[6] Ongun, M. Y. "The Laplace Adomian Decomposition method for solving a model for HIV infection of CD4 ${ }^{+} \mathrm{T}$ cells." Mathematical and Computer Modelling 53, no. 5-6(2011): 597-603.

[7] Merdan, M., Gökdoğan, A., andYildirim, A. "On the numerical solution of the model for HIV infection of CD4 ${ }^{+} \mathrm{T}$ cells." Computers and Mathematics with Applications 62, no. 1(2011): 118-123.

[8] Yüzbaş1, Ş. "A numerical approach to solve the model for HIV infection of $\mathrm{CD}^{+} \mathrm{T}$ cells." Applied Mathematical Modelling 36, no. 12(2012): 5876-5890.

[9] Srivastava, V. K., Awasthi, M. K., and Kumar, S. "Numerical approximation for HIV infection of CD4 ${ }^{+} \mathrm{T}$ cells mathematical model." Ain Shams Engineering Journal 5, no. 2(2014): 625-629.

[10] Dogan, N. "Numerical treatment of the model for HIV infection of $\mathrm{CD}^{+} \mathrm{T}$ cells by using multistep Laplace Adomian decomposition method." Discrete Dynamics in Nature and Society2012, no. 976352 (2012): 1-11.

[11] Khalid, M., Sultana, M., Zaidi, F., and Khan, F. S. "A numerical solution of a model for HIV infection CD4 ${ }^{+}$T-Cell."International Journal of Innovation and Scientific Research 16,(2015): 79-85.

[12] Sabermahani, S., and Ordokhani, Y. (2019). Bernoulli collocation method for solving a model of HIV infection of $C D 4^{+} T$ cells. In $4^{\text {th }}$ International Conference on Combinatorics, Crytography, Computer Science and Computing (pp. 342-348).

[13] Raja, M. A. Z., Asma, K., and Aslam, M. S. "Bio-inspired computational heuristics to study models of HIV infection of $\mathrm{CD} 4^{+}$T-cell."International Journal of Biomathematics 11, no. 02(2018): 1850019-1-1850019-32.

[14] Mirzaee, F., andSamadyar, N. "Parameters estimation of HIV infection model of $\mathrm{CD} 4^{+} \mathrm{T}-$-cells by applying orthonormal Bernstein collocation method." International Journal of Biomathematics 11, no. 02(2018): 1850020-1-1850020-19.

[15] Noeiaghdam, S., and Ghiasi, E. K. "Solving a non-linear model of HIV infection for $\mathrm{CD} 4^{+} \mathrm{T}$ cells by combining Laplace transformation and Homotopy analysis method."arXiv e-prints arXiv:1809.06232, (2018): 1-25.

[16] Rufai, M. A., Duromola, M. K., and Ganiyu, A. A. "Derivation of one-sixth hybrid block method for solving general first order ordinary differential equations." IOSR Journal of Mathematics 12,(2016): 20-27.

[17] Omar, Z., and Adeyeye, O. "Numerical solution of first order initial value problems using a self-starting implicit two-step Obrechkoff-type block method." Journal of Mathematics and Statistics 12, no. 2(2016): 127-134.

[18] Sunday, J., Skwame, Y., and Tumba, P. "A quarter-step hybrid block method for first-order ordinary differential equations." Journal of Advances in Mathematics and Computer Science 6, no. 4 (2015): 269-278.

[19] Akinfenwa, O. A., Akinnukawe, B., and Mudasiru, S. B. "A family of continuous third derivative block methods for solving stiff systems of first order ordinary differential equations." Journal of the Nigerian Mathematical Society 34, no. 2(2015): 160-168.

[20] Zabidi, M. Z. M., Majid, Z. A., and Senu, N. "Solving stiff differential equations using A-stable block method." International Journal of Pure and Applied Mathematics 93, no. 3(2014): 409-425.

[21] Sunday, J., Odekunle, M. R., and Adesanya, A. O. "Order six block integrator for the solution of first order ordinary differential equations." International Journal of Mathematics and Soft Computing3, no. 1(2013): 87-96.

[22] Areo, E. A., and Adeniyi, R. B. "Sixth-order hybrid block method for the numerical solution of first order initial value problems." Mathematical Theory and Modeling 3, no. 8(2013): 113-120

[23] Omar, Z. B., and Suleiman, M. "Solving first order systems of ordinary differential equations using parallel R-point block method of variable step size and order." Chiang Mai Journal of Science 37,(2010): 1-13.

[24] Aksah, S. J., and Ibrahim, Z. B. "Singly diagonally implicit block backward differentiation formulas for HIV infection of CD4+T cells." Symmetry 11, no. 5,625(2019): 1-8.

[25] Adeyeye, O. (2017). Generalised k-step block methods for the direct solution of higher order ordinary differential equations. PhD Thesis, Universiti Utara Malaysia.

[26] Fatunla, S. O. (1988). Numerical methods for initial value problems in ordinary differential equation. Academic Press, New York.

[27] Butcher, J. C. (2008). Numerical methods for ordinary differential equations (second edition). West Sussex: Wiley.

[28] Hassani, H., Mehrabi, S., Naraghirad, E., Naghmachi, M., and Yüzbaşi, S. "An optimization method based on the generalized polynomials for a model of HIV Infection of CD4 ${ }^{+}$T-Cells." Iranian Journal of Science and Technology, Transactions A: Science,(2020): 1-10.

[29] Shanmugasundaram, N., Ganesh, E.N., Kumar, N. "Estimation of power analysis in WLAN infrastructure" International Journal of Engineering and Technology(UAE), 2018, 7(2), pp. 198-200

[30] Shanmugasundaram, N., Sushita, K., Kumar, S.P., Ganesh, E.N. "Genetic algorithm-based road network design for optimising the vehicle travel distance" International Journal of Vehicle Information and Communication Systems, 2019, 4(4), pp. 355-374 